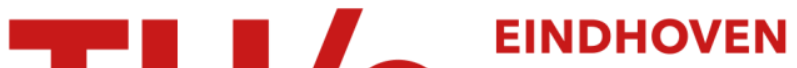 UNIVERSITY OF TECHNOLOGY
}

\section{Spreading of a wetting film under the action of van der Waals forces}

Citation for published version (APA):

Pismen, L. M., Rubinstein, B. Y., \& Bajlekov, I. B. (2000). Spreading of a wetting film under the action of van der Waals forces. Physics of Fluids, 12(3), 480-483. https://doi.org/10.1063/1.870253

DOI:

10.1063/1.870253

Document status and date:

Published: 01/01/2000

\section{Document Version:}

Publisher's PDF, also known as Version of Record (includes final page, issue and volume numbers)

\section{Please check the document version of this publication:}

- A submitted manuscript is the version of the article upon submission and before peer-review. There can be important differences between the submitted version and the official published version of record. People interested in the research are advised to contact the author for the final version of the publication, or visit the $\mathrm{DOI}$ to the publisher's website.

- The final author version and the galley proof are versions of the publication after peer review.

- The final published version features the final layout of the paper including the volume, issue and page numbers.

Link to publication

\section{General rights}

Copyright and moral rights for the publications made accessible in the public portal are retained by the authors and/or other copyright owners and it is a condition of accessing publications that users recognise and abide by the legal requirements associated with these rights.

- Users may download and print one copy of any publication from the public portal for the purpose of private study or research.

- You may not further distribute the material or use it for any profit-making activity or commercial gain

- You may freely distribute the URL identifying the publication in the public portal.

If the publication is distributed under the terms of Article 25fa of the Dutch Copyright Act, indicated by the "Taverne" license above, please follow below link for the End User Agreement:

www.tue.nl/taverne

Take down policy

If you believe that this document breaches copyright please contact us at:

openaccess@tue.nl

providing details and we will investigate your claim. 


\title{
Spreading of a wetting film under the action of van der Waals forces
}

\author{
Len M. Pismen and Boris Y. Rubinstein \\ Department of Chemical Engineering, Technion-Israel Institute of Technology, Haifa 32000, Israel \\ Ivan Bazhlekov \\ Faculty of Mechanical Engineering, Eindhoven University of Technology, P.O. Box 513, 5600 MB Eindhoven, \\ The Netherlands
}

(Received 8 January 1999; accepted 22 October 1999)

\begin{abstract}
The profiles of a spreading wetting film are computed using a variable grid implicit scheme. The form of Tanner's law is deduced from the scaling, and the dependence of its coefficient on ratio of the van der Waals to capillary length and on the inclination angle is determined. (c) 2000 American Institute of Physics. [S1070-6631(00)01502-6]
\end{abstract}

\section{INTRODUCTION}

It has been long recognized that liquids can spread under the action of disjoining pressure. ${ }^{1,2}$ In the case of a liquid attracted to the solid by van der Waals forces, a precursor film is formed, and there is no well-defined contact line that would give rise to the infamous viscous stress singularity., 3 Nevertheless, the problem remains difficult, due to the divergence of both viscous and disjoining pressure terms (which have to compensate each other when the layer thickness tends to zero) and a wide separation between the nearly molecular scale of van der Waals forces and macroscopic scales, where gravity and other extrinsic factors become important. It turns out that the behavior on both scales is interrelated, so that weak forces acting at macroscopic distances strongly influence the shape of a moving film, even close to the transition region between the precursor and the macroscopic film, affecting the visible contact angle. This necessitates matching between computations carried out in the "outer" (gravity) and "inner" (van der Waals) regions and makes the results sensitive to the matching conditions.

After formulating the basic equations in lubrication approximation in Sec. II, we shall stress in Sec. III the pitfalls of this sensitive problem: instability of the static solution and a rescaling symmetry that disallows using the drift velocity as a perturbation parameter. We shall further demonstrate that the familiar cubic-root dependence of the dynamic contact angle on the drift velocity is a direct consequence of the scaling invariance. On the other hand, attempts at computing the proportionality constant in this dependence by solving the stationary equation satisfying asymptotic conditions in the comoving frame are hindered by numerical instabilities, and the "outer" asymptotics converges too slowly to allow effective separation of the inner region, where the van der Waals interactions are essential, and the outer region, where macroscopic factors, like gravity, come into play. This persuades us to seek a numerical solution of the problem using a variable grid extending over widely separated scales and encompassing both regions. The numerical procedure is described in Sec. IV, followed by the discussion of results.

\section{BASIC EQUATIONS AND SCALING}

Consider a liquid film of thickness $h(x)$ resting on a solid plate. It is assumed that the surface inclination is small $\left[h^{\prime}(x)=O(\epsilon) \ll 1\right]$, so that the lubrication approximation is applicable. The general form of an evolution equation in the lubrication approximation (excluding tangential surface stresses) is ${ }^{5}$

$$
h_{t}=\mu^{-1} \nabla \cdot\left(\frac{1}{3} h^{3} \nabla \mathcal{P}\right) \text {. }
$$

The effective pressure $\mathcal{P}$, taking into account surface tension, gravity, and disjoining pressure, is expressed as

$$
\mathcal{P}=-\sigma \epsilon^{2} \nabla^{2} h+\rho g(h-\alpha x)-\frac{A}{6 \pi h^{3}},
$$

where $\sigma$ is surface tension, $\rho$ is density, $g$ is acceleration of gravity, $\epsilon \alpha$ is the inclination angle of the solid surface, and $A$ is the Hamaker constant. We shall assume $A>0$, which corresponds to the case of complete wetting; under these conditions, the liquid is attracted to the solid, and the disjoining pressure is negative. As a result, the liquid layer tends to thicken, or, in other words, the liquid interface is repelled from the solid. Surface tension is commonly presumed to be of $O\left(\epsilon^{-2}\right)$ to make it on par with other forces when the interface is weakly curved; thus we set $\sigma=\sigma_{0} \epsilon^{-2}$.

The characteristic length scales associated with different terms in the evolution equation (1) are widely separated. Choosing a length scale $\lambda$ (as yet not specified) and a suitable time scale $t_{0}=3 \lambda \mu / \sigma_{0}$, Eq. (1) is rewritten in the dimensionless form

$$
h_{t}=-\boldsymbol{\nabla} \cdot\left[h^{3} \nabla\left(\nabla^{2} h+\frac{V}{h^{3}}-G(h-\alpha x)\right)\right],
$$

where 


$$
V=\frac{A}{6 \pi \lambda^{2} \sigma_{0}}, \quad G=\frac{\rho g \lambda^{2}}{\sigma_{0}} .
$$

The length scale in the microscopic boundary layer where intermolecular forces are important is defined by balancing the van der Waals and surface tension terms in Eq. (2). The respective van der Waals length $\lambda^{2}=A /\left(6 \pi \sigma_{0}\right)$ typically falls into a nanoscale range. The gravity parameter based on this length, $G=\rho g A /\left(6 g p \sigma_{0}^{2}\right)$ is quite different from the standard Bond number, and, to avoid misunderstanding, we shall call it the van der Waals number. This parameter, equal to the squared ratio of the van der Waals to capillary length, is very small, and gravity can be neglected in a thin precursor film adjacent to the solid surface. We rewrite the truncated equation valid in this region in the frame moving with the contact line drift velocity $U$ (scaled by $U_{0}=3 \mu / \sigma_{0}$ ). The velocity is assumed to be positive when the bulk fluid layer, situated at $x>0$, advances to the left. After integrating once and setting the integration constant to zero to satisfy the condition of zero net flux through the contact line, this equation becomes

$$
\frac{U}{h^{2}}+\frac{d}{d x}\left(h^{\prime \prime}(x)+\frac{1}{h^{3}}\right)=0 .
$$

Setting here $h^{\prime}(x)=\theta(h)=\sqrt{y(h)}$ yields

$$
\frac{2 U}{h^{2} y^{1 / 2}}+\frac{d}{d h}\left(y^{\prime}(h)+\frac{2}{h^{3}}\right)=0
$$

The static equation at $U=0$ is invariant to the rescaling that changes the slope,

$$
x \rightarrow x / C, \quad h \rightarrow h / \sqrt{C}, \quad y \rightarrow C y .
$$

The factor $C$ is arbitrary, and the rescaling symmetry exists because no forces capable to fix the slope are present in this approximation. The static solution, immediately following from Eq. (5), is $y=h^{-2}+C$, which can be further integrated to

$$
h=\sqrt{C x^{2}-C^{-1}} .
$$

This solution has a zero corresponding to a "contact line" located at $x=C$, and the "true" contact angle is $\pi / 2$. The asymptotic slope $h^{\prime}(x)=\sqrt{y} \asymp C$ was identified by de Gennes et al., ${ }^{6}$ with the macroscopic equilibrium contact angle, but, in fact, it remains indefinite and is not related to any material properties.

\section{ASYMPTOTIC ANALYSIS}

de Gennes et al. ${ }^{6}$ used the static solution (7) as a zero approximation for a solution at $U \ll 1$. This approach is unsuitable for two reasons. First, the static solution (7) is unstable, as any perturbation at the foot of the film would tend to spread further into a layer of minimal (molecular) thickness. Second, velocity never can be treated as a small perturbation, since it can be eliminated from Eq. (4) or (5) by applying the transformation (6) with the scaling factor $C$ $=U^{2 / 3}$. Thus, the well-known Tanner's $\operatorname{law}^{7} \theta=\sqrt{y} \propto U^{1 / 3}$ is a direct consequence of the rescaling invariance. The consequence is that an arbitrarily small velocity causes a finite deviation from the static solution at sufficiently large distances, and a regular perturbation solution at $U \ll 1$ is meaningless.

A correct asymptotics of Eq. (4) at both small and large $h$ was obtained in an earlier work by de Gennes. ${ }^{2,8}$ At $h$ $\rightarrow 0$, the surface tension is negligible, and the viscous stress is balanced by disjoining pressure, contrary to a spurious balance between surface tension and disjoining pressure in the static solution (7). This yields

$$
h^{\prime}(x)=\sqrt{y_{0}} \asymp \frac{1}{3} U h^{2}, \quad h \asymp-3 / x, \quad \text { at } h \rightarrow 0, \quad x \rightarrow-\infty,
$$

valid up to a molecular cutoff of $h$.

On the other hand, at $h \gg 1$, or large positive $x$, the disjoining pressure is negligible, and the balance between viscosity and surface tension leads to the asymptotics

$$
y \asymp\left(3 U \ln \frac{h}{h_{0}}\right)^{2 / 3}, \quad h \asymp x\left(3 U \ln \frac{h}{h_{0}}\right)^{1 / 3}, \quad \text { at } x, h \rightarrow \infty,
$$

where $h_{0}$ is an indefinite constant. This asymptotics is valid only when $h$ is logarithmically large.

Hervet and de Gennes ${ }^{8}$ computed the constant $h_{0} \approx 2.5$ by integrating Eq. (4), starting from the asymptotics at $h$ $\rightarrow 0$. For this purpose, the latter had to be made more precise. Setting $y=y_{0}+\delta y_{1}$, where $y_{0}$ is defined by Eq. (8) and $\delta$ is a bookkeeping small parameter, and neglecting terms in Eq. (5) vanishing at $h \rightarrow 0$ yields the equation for the asymptotic correction $y_{1}$,

$$
y_{1}^{\prime \prime}(h)-27 h^{-8} y_{1}=0,
$$

where $U$ has been rescaled to unity. The solution is a modified Bessel function $\sqrt{h} K_{1 / 6}\left(\sqrt{3} h^{-3}\right)$, which can be replaced by its asymptotics $h^{2} \exp \left(-\sqrt{3} h^{-3}\right)$ multiplied by an arbitrary constant. This constant should be adjusted to ensure that integration of Eq. (5) leads to a finite value of $y(h)$ at large $h$. We found the result to be very sensitive to the choice of an initial point and could not reliably confirm the numerical value of $h_{0}$ reported by Hervet and de Gennes. ${ }^{8}$

Several authors ${ }^{9-11}$ attempted to describe the macroscopic spreading process under the action of either gravity, or surface tension, or both, assuming a self-similar form of the solution in the macroscopic region, while accounting for the effect of disjoining pressure through matching with de Gennes' outer asymptotics. The matching could not be, however, exact, due to the incompatibility of asymptotics and the breakdown of self-similarity.

Hocking ${ }^{12}$ overcame this handicap by introducing an intermediate region where a rescaled form of Eq. (3) with $G$ $=0$ was solved numerically. The numerical solution could not, however, be matched with the short-time solution.

\section{NUMERICAL SOLUTION}

In view of the difficulties arising in numerically assisted intermediate asymptotics and matching, we undertook to obtain a numerical solution directly using a variable grid to extend the integration to a large region. We addressed specifically the problem of a dynamic meniscus on a moving 
(a)

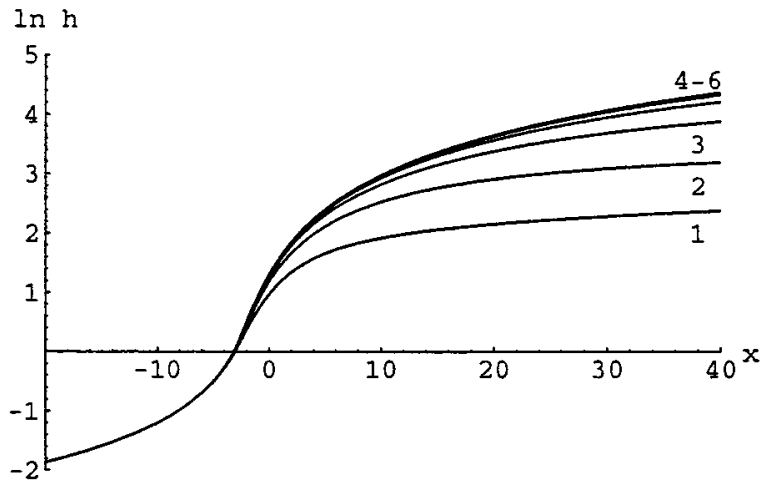

(b)

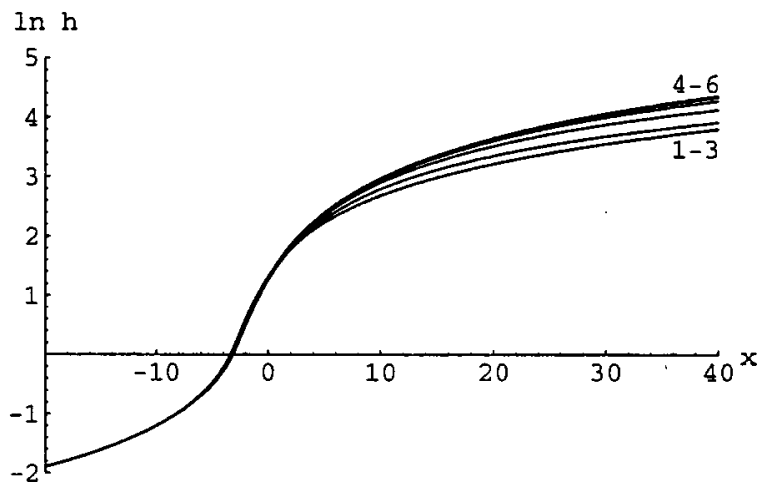

FIG. 1. Logarithmically scaled profiles $\ln h(x)$ at $\alpha \rightarrow 0$ (a) and $\alpha=1$ (b) calculated for different values of the rescaled van der Waals number $G$. The values of $\log G^{-1}$ are shown below the corresponding curves; closely lying curves are marked by the lower and upper applicable values of $\log G^{-1}$.

inclined support, which admits stationary solutions, and solved the stationary form of Eq. (1) describing the profile of an infinitely extended film on an inclined plane in the frame moving with the velocity $U$ :

$$
\frac{1}{h^{2}}+\frac{d}{d x}\left(h^{\prime \prime}(x)+\frac{1}{h^{3}}-G h\right)+G \alpha=0,
$$

where $U$ has been eliminated by rescaling the variables and parameters as

$$
x \rightarrow x U^{-2 / 3}, \quad h \rightarrow h U^{-1 / 3}, \quad G \rightarrow G U^{4 / 3}, \quad \alpha \rightarrow \alpha U^{1 / 3} .
$$

The boundary conditions are given by (8) and

$$
h^{\prime}(x)=\alpha, \quad \text { at } x \rightarrow \infty .
$$

Several alternative numerical methods were tried in order to choose the most reliable algorithm. Explicit schemes proved to be very unstable at physically relevant small values of $G$. We switched therefore to a more stable implicit scheme. In order to achieve good accuracy in the most interesting region of transition from a precursor film to bulk liquid where the profile changes sharply, while keeping the time and memory requirements reasonable, we used a nonuniform grid consisting of five segments. The first and the last segments had a fixed large step size, the middle segment

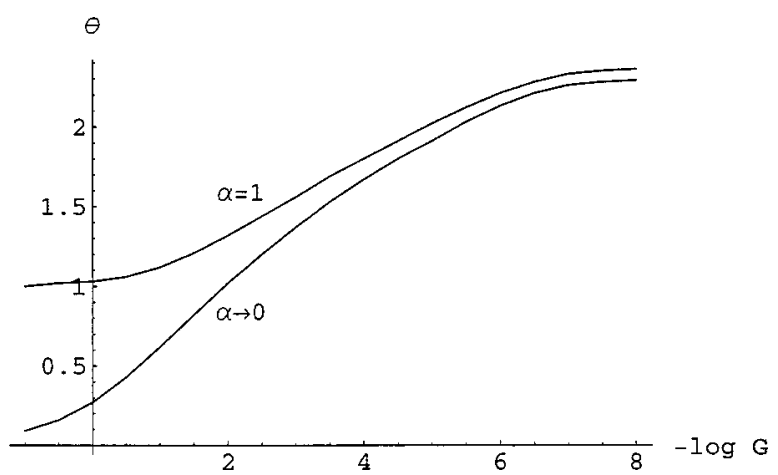

FIG. 2. The contact angle $\theta$, defined as the inclination at the inflection point, calculated for $\alpha \rightarrow 0$ (lower curve) and $\alpha=1$ (upper curve) and different values of rescaled van der Waals number $G$. had a very fine resolution, also with a fixed step, and two intermediate segments had a variable grid enabling a gradual transition between the fixed step segments. The accuracy of calculations was increased by switching to the six-points derivatives approximation.

The numerical scheme used in calculations is fully implicit. We need to find the solution of a stationary problem,

$$
F\left[x, h(x), h^{\prime}(x), h^{\prime \prime}(x), \ldots\right]=0,
$$

which is found by introducing some artificial time $\xi$ and applying a relaxation technique. Then the problem to be solved is rewritten as

$$
\begin{aligned}
& \partial F^{(n+1)}\left[x, h(x, \xi), h_{x}^{\prime}(x, \xi), h_{x x}^{\prime \prime}(x, \xi), \ldots\right] / \partial \xi \\
& \quad=-F^{(n)}\left[x, h(x, \xi), h_{x}^{\prime}(x, \xi), h_{x x}^{\prime \prime}(x, \xi), \ldots\right],
\end{aligned}
$$

where the superscript denotes a "time" step.

Denoting $R(x)=\partial h(x, \xi) / \partial \xi$, and, correspondingly, $R^{\prime}(x)=\partial h_{x}^{\prime}(x, \xi) / \partial \xi, R^{\prime \prime}(x)=\partial h_{x x}^{\prime \prime}(x, \xi) / \partial \xi, \ldots$, we can express the problem in the form

$$
\frac{\partial F^{(n+1)}}{\partial h} R+\frac{\partial F^{(n+1)}}{\partial h^{\prime}} R^{\prime}+\frac{\partial F^{(n+1)}}{\partial h^{\prime \prime}} R^{\prime \prime}+\cdots=-F^{(n)} .
$$

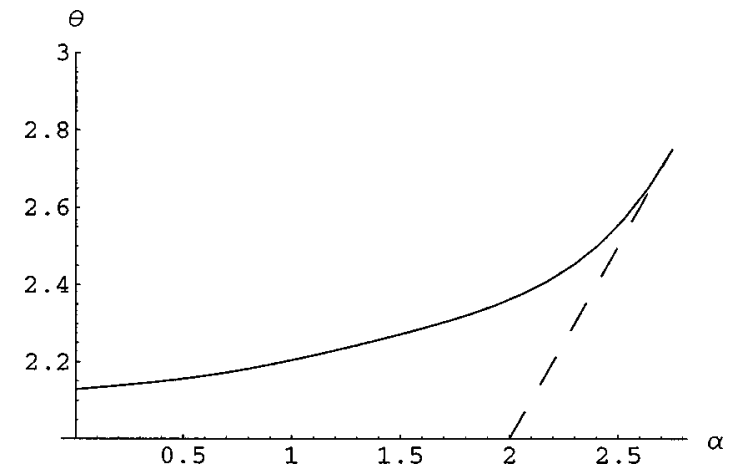

FIG. 3. Dependence of the computed contact angle on the inclination angle $\alpha$ at rescaled van der Waals number $G=10^{-6}$. The limiting case $\theta=\alpha$ is shown by the dashed line. 
Using the discretization for $R, R^{\prime}, R^{\prime \prime}, \ldots$, with respect to a chosen multipoint scheme on a specified grid, we rewrite the problem in the matrix form for the vector $R(i)$, where $i$ denotes the tag of a grid point.

The boundary conditions are incorporated into the above numerical scheme in a similar way. The function values and their derivatives at both ends of the integration interval are defined with the help of additional points $x(-1), x(-2)$ and $x(N+1), x(N+2)$, respectively, at the left and right end. For example, the left asymptotic condition $h \asymp-3 / x$ is coded as follows. It is assumed that this condition is satisfied at the leftmost point of the region, as well as at the two extra points: $h(i)=-3 / x(i), i=-2,-1,0$, or $G_{l}(x, h) \equiv h(i)$ $+3 / x(i)=0$. Then

$$
d G_{l}(h) / \partial \xi=R(i)=-h(i)-3 / x(i),
$$

and for $i=0,-1,-2$ we get $R(i)=0$ automatically, so that there are no additional terms in the matrix for the first two rows.

Similarly, at the right end $(i=N)$ we need to satisfy the condition $G_{r}\left(h^{\prime}\right) \equiv h^{\prime}(x)-\alpha=0$, which produces $R^{\prime}(i)=0$, or $R(i+1)=R(i-1)$ for $i=N, N+1$. The last relation is used for tuning the elements of the $N \times N$ matrix used in the calculation process. In this way, we ensure that the correct asymptotics is reached at the boundary of a large but finite integration interval.

Since a computation using a fully implicit scheme is a generalization of the Newton method for a large set of algebraic equations, the stability of computations is very sensitive to the choice of an initial approximation. We found that the computations became unstable as the value of $G$ decreased. Therefore we started the calculations with a very large value $G=10$ and an initial profile satisfying the specified boundary conditions. With a properly chosen initial profile, the iteration process converged successfully. Then the value of $G$ was reduced by a certain decrement and the iteration process was repeated with the solution obtained at the preceding (larger) value of $G$ used as the initial profile. In this way, $G$ was gradually reduced to physically relevant small values. In a similar way, we gradually reduced the inclination angle $\alpha$ to obtain film profiles at $\alpha \rightarrow 0$. The limit $\alpha=0$ is, of course, singular, as no stationary meniscus exists in this case.

\section{RESULTS AND CONCLUSIONS}

Figure 1 shows logarithmically scaled profiles at $\alpha \rightarrow 0$ and $\alpha=1$ in the region close to the origin where the steepest change of the thickness is observed. One can clearly see that all profiles approach a common asymptotic form at small $x$, but digress at the right, depending on $G$. The curves at small values of $G$ that lie close to one another in this region digress at larger distances. Although the limit of small $\alpha$ is singular, it is interesting because the distinction among various curves is most pronounced in this limit (it is also most difficult to compute).
The "visible" contact angle cannot be defined in this case unequivocally, as the slope changes continuously. We chose to characterize the different profiles by the inclination angle calculated at the inflection point. The dependence of the "contact angle" defined in this way on the van der Waals number is shown in Fig. 2. It has to be reminded that the computed slopes should be multiplied, according to Eq. (12), by $U^{1 / 3}$, so that the data show, in fact, the change of the coefficient in Tanner's law.

The dependence of the "contact angle" on the inclination angle $\alpha$ is shown in Fig. 3. Although the film profile is indeed universal (in the rescaled units) at $G \ll 1$ and moderate $h$, it is never approximated by the intermediate asymptotics (9), since gravity becomes important before the thickness becomes logarithmically large.

Qualitative considerations make it obvious that the interface, being repelled from the solid, is stable in the wetting case. Instabilities in the spanwise direction may develop in flow down an inclined plane or due to the Marangoni effect, in the presence of either a longitudinal or transverse temperature gradient. The numerical solution obtained above can be used as a basic state in the numerical stability analysis, which will be the subject of a forthcoming communication. $^{13}$

\section{ACKNOWLEDGMENTS}

This research has been supported by the Israel Science Foundation. I. B. acknowledges travel support by the Minerva Center for Nonlinear Physics of Complex Systems, which made this cooperation possible.

${ }^{1}$ B. V. Derjaguin, N. V. Churaev, and V. M. Muller, Surface Forces (Consultants Bureau, New York, 1987).

${ }^{2}$ P. G. de Gennes, "Wetting: statistics and dynamics," Rev. Mod. Phys. 57, 827 (1985).

${ }^{3}$ C. Huh and L. E. Scriven, "Hydrodynamic model of steady movement of a solid/liquid/fluid contact line,' J. Colloid Interface Sci. 35, 85 (1971).

${ }^{4}$ E. B. Dussan V, " On the spreading of liquids on solid surfaces: static and dynamic contact lines," Annu. Rev. Fluid Mech. 11, 371 (1979).

${ }^{5}$ A. Oron, S. G. Bankoff, and S. H. Davis, "Long-scale evolution of thin liquid films,"' Rev. Mod. Phys. 69, 931 (1997).

${ }^{6}$ P. G. de Gennes, X. Hue, and P. Levinson, "Dynamics of wetting: local contact angles,'” J. Fluid Mech. 212, 55 (1990).

${ }^{7}$ L. Tanner, "The spreading of silicone oil on horizontal surfaces," J. Phys. D 12, 1473 (1979). The original Tanner's law refered to an increase of the radius of a spreading drop proportionally to $t^{1 / 10}$, but de Gennes ${ }^{2}$ extended the term to the $\theta(U)$ dependence at the root of this spreading law.

${ }^{8}$ H. Hervet and P. G. de Gennes, "The dynamics of wetting: precursor films in the wetting of 'dry' solids," C. R. Acad. Sci., Ser. II: Mec., Phys., Chim., Sci. Terre Univers 299, II 499 (1984).

${ }^{9}$ R. Chebbi and M. S. Selim, "Capillary spreading of liquid drops on solid surface," J. Colloid Interface Sci. 195, 66 (1997).

${ }^{10}$ V. M. Starov, V. V. Kalinin, and J. D. Chen, "Spreading of liquid drops over dry surfaces," Adv. Colloid Interface Sci. 50, 187 (1994).

${ }^{11}$ C. Treviño, C. Ferro-Fontán, and F. Méndez, "Asymptotic analysis of axisymmetric drop spreading," Phys. Rev. E 58, 4478 (1998).

${ }^{12}$ L. M. Hocking, "The wetting of a plane surface by a fluid," Phys. Fluids 7, 1214 (1995).

${ }^{13}$ A. A. Golovin, B. Y. Rubinstein, and L. M. Pismen, in preparation. 